\title{
Análise de um modelo preditivo para o diagnóstico de alergia a fármacos baseado na história clínica
}

\section{Analysis of a predictive model for the diagnosis of allergic drug reactions based on the medical history}

Data de receção / Received in: 30/12/2018

Rev Port Imunoalergologia $2020 ; 28$ (I): I I-I 7

Bárbara Kong Cardoso, Sofia Farinha, Marta Martins, Elza Tomaz, Filipe Inácio

Serviço de Imunoalergologia - Centro Hospitalar de Setúbal

\section{RESUMO}

Fundamentos: As reações alérgicas a fármacos constituem um importante problema de saúde pública associado a uma morbilidade e mortalidade significativas. Sendo o estudo alergológico nestas situações consumidor de tempo e recursos consideráveis, um modelo preditivo que permitisse, com base nos dados clínicos, avaliar o risco de diagnóstico positivo de uma forma objetiva, poderia simplificar este estudo. Objetivo: Testar o modelo preditivo construído por Hierro Santorino em doentes seguidos no nosso Serviço de Imunoalergologia por suspeita de alergia medicamentosa e verificar o seu desempenho nesta população. Métodos: Foram avaliados os processos clínicos dos doentes que recorreram à consulta de Imunoalergologia por suspeita de alergia medicamentosa com estudo alergológico concluído entre janeiro de 2017 e junho de 2018. Foram registados os dados identificados como fatores preditivos no referido modelo, assim como o resultado do estudo alergológico. Foi aplicado o algoritmo proposto por Hierro Santorino a esta população e verificado o seu desempenho. Resultados: Foram analisados 159 casos correspondendo a 143 doentes, 54 (37,8\%) do sexo feminino e com uma média de idade 42, I $\pm 25,4$ anos. Em 108 casos havia apenas um fármaco suspeito. Em $39 \%$ dos casos, os antibióticos betalactâmicos foram os fármacos suspeitos, em $31 \%$ os antinflamatórios não esteroides (AINEs) e em 29,6\% foram suspeitos fármacos de outras classes. Em 18,2\% dos casos foi feito o diagnóstico de alergia, em 15,7\% de intolerância a AINEs, em 5\% de intolerância a inibidores da enzima conversora da angiotensina e em $54,1 \%$ dos casos foi excluída alergia. Quando foi aplicado o modelo em estudo a probabilidade média de diagnóstico positivo calculada para o grupo dos alérgicos foi de $73,3 \%$ (28,6\% a 91,7\%) e de $68,7 \%$ 
(8,4 a 96,4\%) para o grupo dos não alérgicos, não se encontrando um limiar com bom poder discriminativo entre as duas situações (curva ROC). Conclusões: $O$ modelo testado revelou um mau desempenho nesta população, sugerindo que os modelos preditivos necessitam de aperfeiçoamento para virem a ser uma ferramenta útil no estudo alergológico das reações a fármacos.

Palavras-chave: Alergia a fármacos, hipersensibilidade a fármacos, estudo alergológico, modelos preditivos, algoritmos de decisão.

\section{ABSTRACT}

Background: Allergic drug reactions represent an important public health problem associated with a significant mortality and morbidity. The allergy workup in this situation is expensive and time consuming. A predictive model using medical history would allow us to assess the risk of a positive result and might simplify the diagnostic process. Aims: To assess the performance of the Hierro Santorino predictive model in patients from our Immunoallergology department investigated for drug allergy. Methods: We included in our study patients referred to our department for drug allergy whose investigation was concluded between January 2017 and June 2018. We collected clinical data identified as the predictive factors in the mentioned model, as well as the results from the allergy work up. The Hierro Santorino model was then applied to our population and its performance was evaluated. Results: We analyzed 159 cases corresponding to 143 patients, 54 (37.8\%) females, mean age of $42.1 \pm 25.4$ years. In 108 cases there was only I drug involved. In $39 \%$ of the cases a beta-lactam was the suspected drug, in $31 \%$ a nonsteroidal anti-inflammatory drug (NSAID) and in 29.6\% other drug classes were suspected. In $18.2 \%$ of the cases the final diagnosis was positive for drug allergy, in 15.7\% "NSAID intolerance" was established, 5\% of the patients had "angiotensin-converting-enzyme inhibitor intolerance" and in 54. $\%$ drug allergy was excluded. As the model under study was applied the mean probability of drug allergy was $73.3 \%$ (26.6\% to $91.7 \%$ ) in the allergic group and $68.7 \%$ (8.4\% to $96.4 \%$ ) in the non-allergic group. There was no cut-off value with capacity to discriminate between the outcomes. Conclusions: The model tested in our population revealed a poor performance suggesting that predictive models still need improving to be used as a tool in the allergy workup.

Key-words: Allergic drug reaction, drug hypersensitivity, allergy workup, predictive models, decision-making algorithm.

\section{INTRODUÇÃO}

A $s$ reações adversas a fármacos constituem um importante problema de saúde pública, não só associado a uma morbilidade e mortalidade importante, como também a custos desnecessários'.

A avaliação por Imunoalergologia é crucial e permite a confirmação ou a exclusão de alergia na maioria dos casos. Existem vários meios complementares de diagnóstico disponíveis, porém com uma sensibilidade inferior à desejável. Na maioria dos casos é necessária uma prova de provocação para confirmação ou exclusão do diagnóstico. Esta prova, para além do tempo e custo associados, não está isenta de riscos, mas de facto é necessária, dado que é negativa na maioria dos casos, excluindo alergia ${ }^{2}$. Em qualquer das situações, o estudo alergológico de uma suspeita de alergia a fármacos consome tempo e recursos apreciáveis.

Para otimizar este estudo seria útil dispor de uma ferramenta que permitisse avaliar objetivamente o risco 
de um diagnóstico positivo. Esta avaliação poderia orientar e eventualmente simplificar o processo do estudo.

É este o contexto que justifica a tentativa de construção de modelos preditivos. A utilização destes modelos, que teve as suas origens no setor económico, tem sofrido nos últimos anos uma ampla generalização a quase todas as áreas da atividade humana, incluindo a medicina ${ }^{3}$. Aqui as suas aplicações vão da área da gestão às do diagnóstico e decisão, incluindo decisões de estratégias preventivas e decisões terapêuticas individuais.

Este tipo de modelos tem sido relativamente pouco usado especificamente na Imunoalergologia, mas modelos preditivos da incidência e persistência da rinite ou do eczema atópico na adolescência, bem como modelos preditivos da evolução em alergia alimentar, têm sido construídos e aplicados nos últimos anos ${ }^{4-6}$. Surge, no entanto, a questão da legitimidade da generalização destes modelos, considerando que diferentes características das populações vão condicionar o seu desempenho. Assim, a aplicação de um modelo preditivo exige uma prévia validação do mesmo na população a que vai ser aplicado.

Foi neste contexto que os autores decidiram testar o modelo preditivo criado para o diagnóstico de reações alérgicas a fármacos baseado na história clínica publicado por Hierro Santorino et al. ${ }^{7}$ num grupo de doentes estudados por alergia medicamentosa no nosso Serviço de Imunoalergologia. No referido modelo foi utilizado o método de regressão logística binária simples para relacionar as múltiplas variáveis da anamnese com o diagnóstico final. Os coeficientes dos fatores identificados foram usados na construção de uma fórmula para o cálculo da probabilidade de reação a priori.

\section{OBJETIVO}

Testar o modelo preditivo construído por Beatriz Hierro Santurino et al. ${ }^{7}$ em doentes seguidos no Serviço de Imunoalergologia do nosso hospital por suspeita de alergia medicamentosa e verificar o seu desempenho.

\section{MATERIAL E MÉTODOS}

\section{Desenho do estudo e população selecionada}

O presente estudo foi realizado em duas fases. A primeira consistiu numa avaliação retrospetiva de processos clínicos dos doentes que recorreram à consulta de Imunoalergologia por suspeita de alergia medicamentosa com estudo alergológico concluído entre janeiro de 2017 e junho de 2018.

Foram registados os dados identificados como fatores preditivos no modelo desenvolvido por Hierro Santurino et al. ${ }^{7}$, nomeadamente sexo, idade à data da reação, número de fármacos implicados, classe do fármaco suspeito, número de doses, manifestações clínicas, período de latência entre a exposição e a reação e tempo decorrido até à consulta. Foi ainda registado o resultado do estudo alergológico.

Relativamente às manifestações clínicas, foram utilizados os mesmos critérios usados por Hierro Santurino na construção do seu modelo, nomeadamente sintomas compatíveis com anafilaxia, angioedema, urticária, drug reaction with eosinophilia and systemic symptoms (DRESS), Stevens-Johnson Syndrome, eritema fixo, exantema, eczema, asma ou rinoconjuntivite foram consideradas manifestações "alérgicas". Outras reações foram classificadas como "não alérgicas".

Foram consideradas várias categorias de diagnóstico:

I. Alergia confirmada: clínica comprovada por testes cutâneos ou prova de provocação positivos;

2. Sem alergia (confirmado): quando a prova de provocação foi negativa;

3. Possível alergia: suspeita de acordo com a história clínica, mas sem estudo confirmativo.

Uma vez que a intolerância a antinflamatórios não esteroides (AINEs) e a inibidores da enzima conversora da angiotensina (IECAs) provocam comummente reações de hipersensibilidade que clinicamente se assemelham a reações alérgicas, foram incluídas outras duas categorias: 
4. Diagnóstico ou suspeita de intolerância a AINEs: quando a prova de provocação a aspirina foi positiva ou havia história consistente de reação a pelo menos dois grupos de AINEs;

5. Diagnóstico ou suspeita de intolerância a IECAs: quando suspensão do IECA em uso se relacionou com a cessação dos episódios de angioedema.

A segunda fase consistiu na aplicação do algoritmo proposto por Hierro Santurino à nossa população.

A fórmula usada no cálculo da probabilidade a priori do diagnóstico foi:

I. Ln (odds do diagnóstico) $=-2,533+$ sexo (feminino ou masculino) $+0,024 \times$ idade-0, $145 \times$ n. ${ }^{\circ}$ de fármacos envolvidos + período de latência (imediato, tardio ou desconhecido) + n. ${ }^{\circ}$ de doses (I.a , várias

Quadro I. Modelo preditivo (Hierro Santurino) para um diagnóstico confirmado de alergia

\begin{tabular}{|c|c|}
\hline & Coeficiente \\
\hline Sexo $($ feminino $=1$, masculino $=0$ ) & $-0,226$ \\
\hline Idade à data da reação (anos) & 0,024 \\
\hline N..$^{\circ}$ de fármacos envolvidos & $-0, \mid 45$ \\
\hline $\begin{array}{l}\text { Período de latência } \\
\text { Imediato } \\
\text { Tardio } \\
\text { Desconhecido }\end{array}$ & $\begin{array}{l}1,116 \\
0,758\end{array}$ \\
\hline $\begin{array}{l}\text { N. }{ }^{\circ} \text { de doses } \\
\text { I. }{ }^{\text {a dose }} \\
\text { Várias } \\
\text { Desconhecido }\end{array}$ & $\begin{array}{l}-0,062 \\
-0,972\end{array}$ \\
\hline $\begin{array}{l}\text { Sintomas alérgicos } \\
(\text { Não alérgicos }=1 \text {, alérgicos }=0)\end{array}$ & $-2,213$ \\
\hline $\begin{array}{l}\text { Classe de fármacos } \\
\text { AINEs }{ }^{\mathrm{a}} \\
\text { Antibióticos betalactâmicos } \\
\text { Outros }\end{array}$ & $\begin{array}{l}0,097 \\
1,492\end{array}$ \\
\hline $\begin{array}{l}\text { Tempo até à consulta }(<12 \text { meses }=0 \text {, } \\
>12 \text { meses }=1)\end{array}$ & $-0,732$ \\
\hline Constante & $-2,533$ \\
\hline
\end{tabular}

${ }^{\text {a }}$ AINEs = Anti-inflamatórios não esteroides ou desconhecido) + sintomas alérgicos (sugestivo ou não sugestivo) + classe de fármacos (AINEs, antibióticos betalactâmicos ou outros) + tempo até à consulta ( $>12$ meses ou $<12$ meses);

2. Odds = $\operatorname{Exp}(L n)$;

3. Probabilidade do diagnóstico $=($ Odds $/ 1+O d d s) \times 100$.

Os coeficientes das variáveis utilizadas constam da Quadro I. Foram incluídas na análise as categorias diagnósticas I e 2. Foi utilizado o programa SPSS Statistics, versão 23 .

Os testes cutâneos e as provas de provocação com fármacos foram realizados e interpretados de acordo com - Manual de Boas Práticas em Imunoalergologia ${ }^{8}$ e as recomendações do Grupo de Interesse de Alergia a Fármacos da $\mathrm{EAACl}^{9,10}$.

\section{RESULTADOS}

Foram avaliados 159 casos de suspeita de alergia medicamentosa correspondendo a 143 doentes, $54(37,8)$ do sexo feminino e $89(62,2 \%)$ do masculino, com idades compreendidas entre I e 83 anos e uma média de 42, I \pm 25,4 anos.

Em 108 casos $(67,9 \%)$ havia apenas um fármaco suspeito. Os antibióticos betalactâmicos foram a classe de fármacos suspeito em 60 casos (37,7\%), os AINEs em 5 I (32,1\%) e “outros fármacos" em 48 (30,2\%). Das reações relatadas, 138 casos $(86,8 \%)$ eram compatíveis com reações alérgicas e 21 ( $13,2 \%)$ com reações não alérgicas.

Em 4 I casos $(25,8 \%)$ a reação ocorreu após a administração da primeira dose do fármaco e em 55 casos $(34,6 \%)$ após a administração de mais de uma dose do fármaco. Em 63 casos (39,6\%) o número de doses administradas antes da reação era desconhecido.

Foram estabelecidos 29 (I8,2\%) diagnósticos de alergia a um fármaco e $86(54,1 \%)$ o diagnóstico de não alergia. Os fármacos implicados estão descriminados na Quadro 2. 
Quadro 2. Fármacos suspeitos nas reações alérgicas dos doentes considerados para aplicação do modelo e diagnóstico final

\begin{tabular}{|c|c|c|c|}
\hline Fármacos/classes & Total & Diagnóstico não alergia & Diagnóstico alergia \\
\hline Ácido alendrónico/colecalciferol & I & I & 0 \\
\hline Alopurinol & I & 0 & 1 \\
\hline AINEs $^{\mathrm{a}}$ & 21 & 12 & 9 \\
\hline Anestésicos & 5 & 5 & 0 \\
\hline Anticonvulsivante & $\mathrm{I}$ & 0 & I \\
\hline Antidiabéticos & 2 & 2 & 0 \\
\hline Antieméticos & 2 & 1 & 1 \\
\hline Antifúngico & I & 1 & 0 \\
\hline Betalactâmicos & 60 & 50 & 10 \\
\hline Clopidogrel & 1 & 1 & 0 \\
\hline Corticoides & 4 & 4 & 0 \\
\hline Outros antibióticos & 9 & 4 & 5 \\
\hline Vacina Bexero $^{\circledR}$ & $\mathrm{I}$ & $\mathrm{I}$ & 0 \\
\hline Vários $^{\mathrm{b}}$ & 6 & 4 & 2 \\
\hline Total & 115 & 86 & 29 \\
\hline
\end{tabular}

a AINEs = Antinflamatórios não esteroides; ${ }^{\text {b }}$ Casos em que estavam implicados fármacos de várias classes distintas

Em 25 casos (15,7\%) o diagnóstico final foi de intolerância a AINEs e em 8 (5\%) de intolerância a IECAs. O diagnóstico de possível alergia foi atribuído a II casos $(6,9 \%)$.

O tempo decorrido desde a reação até à consulta foi de mais de 12 meses em 85 casos $(53,5 \%)$ e de menos de 12 meses em 74 casos (46,5\%).

Em 80 casos $(50,3 \%)$ a reação foi imediata, em 68 $(42,8 \%)$ não imediata, e o tempo de latência foi desconhecido em II casos (6,9\%).

No grupo com diagnóstico final de alergia, a probabilidade média calculada foi de $73,3 \%$ (entre $28,6 \%$ e $91,7 \%$ ); no grupo sem alergia a média foi de $68,7 \%$ (entre 8,4 e 96, 4\%) (Figura I).

A curva ROC obtida tem uma área sobre a curva (AUC) de 0,56 (intervalo de confiança de 95\%, 045-067), verificando-se que os valores de sensibilidade se aproximam em toda a curva dos valores do inverso da especificidade (Figura 2).

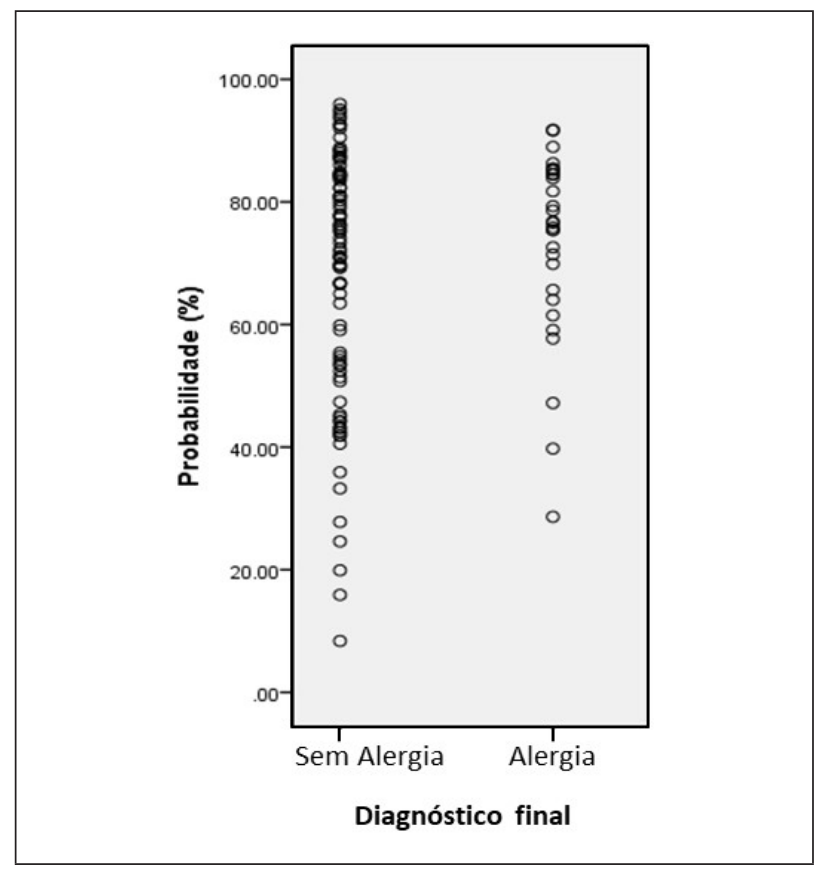

Figura I. Probabilidade a priori / Alergia: Distribuição das probabilidades resultantes da aplicação do modelo pelas categorias de alergia e não alergia 
Bárbara Kong Cardoso, Sofia Farinha, Marta Martins, Elza Tomaz, Filipe Inácio

Quadro 3. Sensibilidade, especificidade, valores preditivos positivo e negativo para os diferentes limiares

\begin{tabular}{|l|c|c|c|c|c|c|c|c|}
\hline Probabilidade limiar & 0,28 & 0,40 & 0,50 & 0,60 & 0,70 & 0,80 & 0,90 & 0,97 \\
\hline Sensibilidade & $\mathrm{I}$ & 0,93 & 0,90 & 0,83 & 0,69 & 0,38 & 0,07 & 0 \\
\hline Especificidade & 0,06 & 0,08 & 0,22 & 0,34 & 0,44 & 0,65 & 0,91 & $\mathrm{I}$ \\
\hline VPP & 0,26 & 0,25 & 0,28 & 0,29 & 0,29 & 0,26 & 0,20 & $\mathrm{I}$ \\
\hline VPN & $\mathrm{I}$ & 0,77 & 0,87 & 0,86 & 0,81 & 0,76 & 0,74 & 0,75 \\
\hline
\end{tabular}

VPP = Valor preditivo positivo; VPN = Valor preditivo negativo

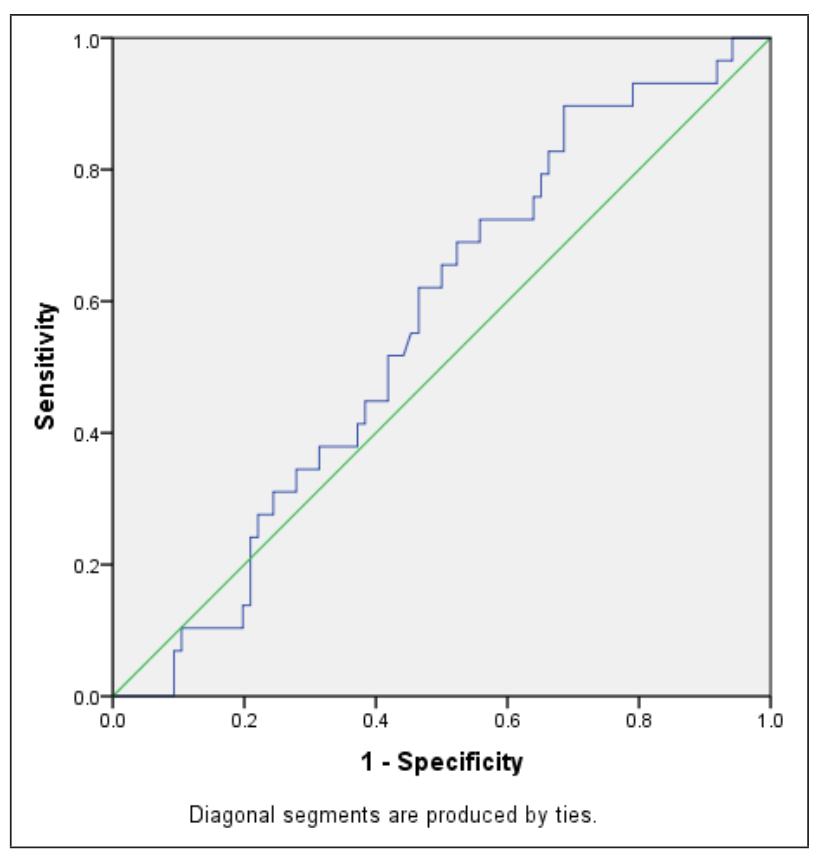

Figura 2. Curva ROC Receiver operating characteristic - Teste da variável "probabilidade" na discriminação do diagnóstico, com linha diagonal de referência

No Quadro 3 estão discriminados os valores de sensibilidade, especificidade, preditivos positivos e negativos para diferentes limiares. Como se pode observar, a probabilidade de reação $28 \%$ é a máxima de $6 \%$ que corresponde a $100 \%$ de sensibilidade, sendo a especificidade correspondente a este limiar. Para atingir os $100 \%$ de especificidade seria necessário considerar - limiar de $96,9 \%$ que corresponde a uma sensibilidade de $0 \%$.

\section{DISCUSSÕES}

O modelo proposto por Hierro Santurino ${ }^{7}$ mostrou um mau desempenho no grupo de doentes aqui estudado, não existindo um valor de probabilidade limiar que permitisse a descriminação entre doentes alérgicos e não alérgicos com um erro aceitável. Deficiências a este modelo tinham já sido apontadas por Esther Moreno et al." ", focando essencialmente aspetos questionáveis da própria construção matemática do algoritmo proposto. Também será de esperar que o peso dos fatores considerados no modelo possa sofrer importantes variações de acordo com as características da população estudada (por exemplo, a prevalência de alergia a fármacos ou a uma classe de fármacos) e com as particularidades do serviço onde é realizado o estudo (por exemplo, tempo de demora da consulta).

Outros modelos na área do diagnóstico da alergia medicamentosa foram desenvolvidos utilizando como fatores preditivos apenas elementos clínicos, nomeadamente no estudo de alergia a antibióticos betalactâmicos. Chiriac et al. ${ }^{12}$ desenvolveram um modelo preditivo utilizando o método de regressão logística multivariada e um outro baseado na construção de uma "árvore de decisão". Os autores concluíram que nenhum deles tinha a capacidade de predizer a alergia a betalactâmicos com precisão e, portanto, de substituir a avaliação alergológica convencional no diagnóstico de alergia a esta classe de fármacos.

Por seu lado, Soria A. et al. ${ }^{13}$ propõem também um algoritmo para a alergia à penicilina baseado em árvores de decisão, que revela um baixo risco de erro na classi- 
ficação, defendendo os autores a sua utilidade para decisões em situações de urgência hospitalar.

O mau desempenho do modelo testado nesta população poderá ser devido a erros na sua construção matemática, mas também a diferentes características da população por nós estudada, por exemplo a prevalências de diagnósticos positivos ou o peso atribuído a determinadas classes de fármacos.

Pensamos que os modelos preditivos poderão ser uma mais-valia na estratificação do risco e servir de base para a otimização de futuros modelos preditivos mais satisfatórios, que possivelmente terão que incluir marcadores genéticos ou outros marcadores biológicos ${ }^{12}$. Parece-nos, no entanto, fundamental a validação do modelo, que deverá ser ensaiada, não só em diferentes contextos geográficos e culturais, como também em diferentes níveis de cuidados de saúde (cuidados de saúde primários ou hospitalares). Estas várias situações poderão implicar modelos diferentes em termos de fatores preditivos identificados, bem como do peso que lhes é atribuído.

\section{CONCLUSÕES}

$\mathrm{Na}$ análise das probabilidades a priori calculadas para os doentes no presente estudo pelo algoritmo de Hierro Santorino, não foi possível encontrar um limiar de probabilidade com bons valores simultaneamente de sensibilidade e especificidade, revelando a incapacidade do modelo na discriminação entre doentes com e sem alergia a um fármaco.

\section{Conflito de interesses}

Os autores declaram que não existem conflitos de interesse.

\section{Contacto:}

Bárbara Kong Cardoso

Serviço de Imunoalergologia, Hospital São Bernardo

R. Camilo Castelo Branco 175

2910-549 Setúbal

Email: barbarakc@gmail.com

\section{REFERÊNCIAS}

I. Manso L, Sánchez-García S, Ruiz-García M, Arochena L, Andregnette V, Fernández-Nieto M, et al. Clinical and socio-economical implications of drug allergy diagnosis in hospitalized patients. J Allergy Clin Immunol 20I0;125(2):ABI58.

2. Messaad D, Sahla H, Benahmed S, Gorard P, Bousquet J, Demoly $\mathrm{P}$. Drug provocation tests in patients with a history suggesting an immediate drug hypersensitivity reaction. World Allergy Organ J 2004;4(I2):257-70.

3. Vogenberg FR. Predictive and prognostic models: Implications for healthcare decision-making in a modern recession. Am Heal DRUG BENEFITS 2009;2(6):218-22.

4. Kellberger J, Dressel H, Vogelberg C, Leupold W, Windstetter $D$, Weinmayr G, et al. Prediction of the incidence and persistence of allergic rhinitis in adolescence: A prospective cohort study. J Allergy Clin Immunol 2012;129(2):397-402.

5. Gustafson E, Pacheco J, Wehbe F, Silverberg J, Thompson W. A machine learning algorithm for identifying atopic dermatitis in adults from electronic health records. IEEE Int Conf Healthc Informatics 2017;2017:83-90.

6. Sugiura S, Matsui T, Nakagawa T, Sasaki K, Nakata J, Kando N, et al. Development of a prediction model of severe reaction in boiled egg challenges. Allergol Int 2016;65(3):293-9.

7. Hierro Santurino B, Mateos Conde J, Cabero Morán MT, Mirón Canelo JA, Armentia Medina A. A predictive model for the diagnosis of allergic drug reactions according to the medical history. J Allergy Clin Immunol Pract 2016;4(2):292-300.

8. Colégio Imunoalergologia - Ordem dos Médicos. Manual de Boas Práticas - Procedimentos diagnóstico / tratamento em imunoalergologia. Site da Ordem dos Médicos. 2011.

9. Brockow K, Garvey LH, Aberer W, Atanaskovic-Markovic M, Barbaud A, Bilo MB, et al. Skin test concentrations for systemically administered drugs - an ENDA/EAACI Drug Allergy Interest Group position paper. Allergy. 2013;68:702-12.

10. Aberer W, Bircher A, Romano A, Blanca M, Campi P, Fernandez $\mathrm{J}$, et al. Drug provocation testing in the diagnosis of drug hypersensitivity reactions: General considerations. Allergy 2003;58:854-63.

II. Moreno E, Muñoz-Bellido FJ, Laffond E, Dávila IJ. Predictive models in drug allergy: An appropriate patient selection is needed. J Allergy Clin Immunol Pract 2016;4(5):1015.

12. Chiriac AM, Wang Y, Schrijvers R, Bousquet PJ, Mura T, Molinari $\mathrm{N}$, et al. Designing predictive models for beta-lactam allergy using the drug allergy and hypersensitivity database. J Allergy Clin Immunol Pract 2018;6(I):139-48.

13. Soria A, Autegarden E, Amsler E, Gaouar H, Vial A, Francès C, et al. A clinical decision-making algorithm for penicillin allergy. Ann Med 2017;49(8):710-7. 